Meta

Journal des traducteurs

Translators' Journal

\title{
De la dérive des continents
}

\section{Alain Morissette}

Volume 20, numéro 4, décembre 1975

URI : https://id.erudit.org/iderudit/003666ar

DOI : https://doi.org/10.7202/003666ar

Aller au sommaire du numéro

Éditeur(s)

Les Presses de l'Université de Montréal

ISSN

0026-0452 (imprimé)

1492-1421 (numérique)

Découvrir la revue

Citer cet article

Morissette, A. (1975). De la dérive des continents. Meta, 20(4), 313-317.

https://doi.org/10.7202/003666ar

Ce document est protégé par la loi sur le droit d'auteur. L’utilisation des services d'Érudit (y compris la reproduction) est assujettie à sa politique d'utilisation que vous pouvez consulter en ligne.

https://apropos.erudit.org/fr/usagers/politique-dutilisation/ 


\section{DE LA DÉRIVE DES CONTINENTS}

$\mathrm{Au}$ cours des dernières années, les revues scientifiques ont fait un très large écho à la relance des sciences de la terre, favorisée par la reprise d'une théorie vieille de 63 ans, la dérive des continents (continental displacement, continental drift, continental migration), énoncée pour la première fois par le météorologiste allemand Alfred Wegener.

Selon cette théorie, la disposition actuelle des continents serait le résultat d'un déplacement progressif amorcé, il y a plusieurs millions d'années par la brusque rupture d'une immense masse monolithique. À partir de données traitées sur ordinateur, il a été possible de reconstituer ce continent original, appelé Pangea (Pangaea) qui devait lui-même se scinder par la suite en deux supercontinents, le Laurasia (Laurasia) et le Gondwana (Gondwanaland), correspondant respectivement à l'hémisphère nord et à l'hémisphère sud.

Étayée aujourd'hui de nombreuses preuves paléontologiques, orogéniques et géophysiques, la théorie de la dérive a franchi le cap de l'approximation pour se hisser au rang des véritables disciplines scientifiques.

Le concept de la tectonique globale (global tectonics) ou tectonique des plaques (plate tectonics) représente la pierre angulaire sur laquelle est bâtie toute la théorie de la dérive des continents. Qu'il me soit permis de faire une brève digression terminologique pour apporter certaines précisions quant à l'emploi de l'expression tectonique globale. L'utilisation du mot global par les auteurs d'expression anglaise, immédiatement transposé en français, risque de faire naître, pour les "rigoristes», une certaine confusion qu'il convient de dissiper. D'après GARGE ${ }^{1}$, global tectonics revêt l'acception suivante :

Tectonics on global scale, such as tectonic processes related to very largescale movement of material within the Earth.

WEBIN 2 donne le sens exact de global dans le contexte qui nous occupe :

Of, relating to, or involving the entire world.

D'autre part, l'adjectif global en français, dérivé du substantif globe, n'en a gardé que le sens figuré :

Qui s'applique à un ensemble, qui est pris en bloc (somme globale) PEROB ${ }^{3}$.

1. Margaret Gary, Glossary of Geology, Washington (D.C.), American Geological Institute, 1972. 2. Webster's Third New International Dictionary, Springfield, G. \& C. Merriam Company,

3. Paul Robert, Le Petit Robert, Paris, Société du Nouveau Littré, 1972. 
L'expression tectonique du globe, sous-entendu du globe terrestre, serait donc plus appropriée que tectonique globale. Toutefois, l'utilisation pléthorique de cette dernière semble avoir atteint un point de non-retour : l'usage a ses raisons que la raison ne connaît point.

La tectonique globale pose comme principe fondamental l'existence d'une mosaïque d'unités qui se comportent, chacune, comme des plaques rigides. Formées aux dorsales médio-océaniques, ces plaques (plates) se développent dans les deux directions perpendiculaires à l'axe des dorsales. Du matériel volcanique nouveau est apporté aux plaques dans les zones d'extension (extension zones), tandis que dans les zones de compression ou zones de subduction (subduction zones), une plaque peut être amenée à s'enfoncer sous une autre et à disparaître ainsi sous le manteau. Une plaque est en quelque sorte une couche de faible vitesse (lowvelocity layer), c'est-à-dire une couche où la vitesse des ondes sismiques est plus faible que dans la couche supérieure. La lithosphère (lithosphere) est composée essentiellement de ces plaques - connues aussi sous le nom de plaques lithosphériques (lithospheric plates, plates of lithosphere) - en perpétuel mouvement les unes par rapport aux autres au-dessus de l'asthénosphère (asthenosphere). Les limites des plaques (plate boundaries, plate junctions) se classent donc en trois catégories : les dorsales médio-océaniques où elles se forment, les systèmes d'arcs et de fossés (trenches) où une plaque disparaît sous une autre en glissant dans l'écorce terrestre, et les failles transformantes où deux plaques se déplacent parallèlement à leur bord.

Malgré toutes les connaissances acquises à ce jour, la cause de la dérive des continents est un point qui reste encore à éclaircir. D'après les recherches en cours, il existerait dans le manteau terrestre (earth's mantle) des courants de convection (convection currents), c'est-à-dire des couches d'une certaine viscosité, qui auraient repoussé les continents de part et d'autre. L'existence de ces courants serait également responsable de l'expansion du fond océanique (ocean-floor spreading) ou expansion du fond des mers (sea-floor spreading), provoquée par la rencontre de deux courants, l'un ascendant, l'autre descendant.

L'hypothèse de l'expansion du fond des mers fut étayée, il y a quelques années, par l'introduction d'un concept nouveau, celui des failles transformantes (transform faults). Les crêtes des dorsales médio-océaniques (mid-oceanic ridges), ces bandes longitudinales qui se développent dans l'océan Atlantique, l'océan Indien et le Pacifique Sud, sont coupées par un grand nombre de failles, appelées zones de fracture (fracture zones). Or, il ne s'agit pas de failles conventionnelles horizontales, mais de failles produites par l'expansion du fond des mers au niveau des dorsales médio-océaniques.

Une autre preuve visant à appuyer le phénomène de l'expansion du fond océanique réside dans l'étude qui a été faite des ares insulaires (island arcs). Les systèmes d'arcs insulaires correspondent aux endroits où une plaque océanique disparaît en s'enfonçant par subduction dans l'écorce terrestre. Il faut noter de plus que les arcs insulaires sont le lieu d'importants phénomènes sismiques.

Le paléomagnétisme a également apporté une contribution à la théorie de la dérive des continents. En effet, la preuve du déplacement des pôles ou déplacement 
polaire (polar wandering, wandering of the poles) fut mise en évidence récemment par des scientifiques qui constatèrent que le champ magnétique terrestre avait subi plusieurs inversions (geomagnetic reversal, field reversal, geomagnetic polarity reversal). Cette idée fut combattue pendant un certain temps par les tenants de l'auto-inversion du magnétisme rémanent (remanent magnetism self-reversal), théorie suivant laquelle certaines roches possèdent la propriété d'acquérir une magnétisation rémanente (remanent magnetization) de direction opposée à celle du champ magnétique extérieur (ambient magnetic field). Des recherches poussées ont prouvé que le champ magnétique terrestre a connu plusieurs périodes de polarité (polarity epochs) au cours des siècles. D'autre part, une hypothèse récente fait état de bosses (bumps), situées à la limite entre le noyau (core) et le manteau, qui pourraient provoquer les inversions du champ géomagnétique.

Même si plusieurs modèles ont été proposés jusqu'à maintenant, la plupart basés sur l'hypothèse de l'existence de cellules convectives (convection cells) dans le manteau, il en est un, dit modèle des panaches (plume model) qui semble vouloir s'imposer. Le manteau serait animé d'un mouvement continuel de convection. Du matériel d'origine profonde remonterait rapidement dans des panaches (plumes) jusqu'à la base de la lithosphère, puis divergerait, à partir du point chaud (hot spot) ainsi créé, dans toutes les directions au sein de l'asthénosphère. Après s'être refroidi, ce matériel retomberait lentement à travers le manteau. À l'endroit où un panache atteint la base d'une plaque lithosphérique, une déformation en dôme (doming) précèderait le percement mécanique et thermique de la plaque. De plus, les chaînes volcaniques et les dorsales asismiques (aseismic ridges) constitueraient les traces volcaniques des panaches (plume traces), c'est-à-dire les traînées laissées par le passage des plaques sur les panaches. Selon cette hypothèse, les panaches seraient le moteur principal de la dérive des continents.

\section{LEXIQUE ANGLAIS-FRANÇAIS}

ambient magnetic field aseismis ridge asthenosphere bump continental displacement continental drift continental migration convection cell convection current

core

doming

earth's mantle extension zone

field reversal

fracture zone geomagnetic polarity reversal geomagnetic reversal global tectonics

\author{
champ magnétique extérieur \\ dorsale asismique \\ asthénosphère \\ bosse \\ dérive des continents \\ voir continental displacement \\ voir continental displacement \\ cellule convective \\ courant de convection \\ noyau \\ déformation en dôme \\ manteau terrestre \\ zone d'extension \\ inversion du champ magnétique terrestre \\ zone de fracture \\ voir field reversal \\ voir field reversal \\ tectonique globale \\ tectonique du globe (terrestre)
}


Gondwanaland

hot spot

island arc

Laurasia

lithosphere

lithospheric plate

low-velocity layer

mid-oceanic ridge

ocean-floor spreading

Pangaea

plate

plate boundary

plate junction

plate of lithosphere

plate tectonics

plume

plume model

plume trace

polarity epoch

polar wandering

remanent magnetism self-reversal

remanent magnetization

sea-floor spreading

subduction zone

transform fault

trench

wandering of the poles

\author{
Gondwana (m.) \\ point chaud \\ are insulaire \\ Laurasia (m.) \\ lithosphère \\ plaque lithosphérique \\ couche de faible vitesse \\ dorsale médio-océanique \\ expansion du fond océanique \\ Pangea ( $m$.) \\ plaque \\ limite des plaques \\ voir plate boundary \\ voir lithospheric plate \\ tectonique des plaques \\ panache \\ modèle des panaches \\ trace volcanique des panaches \\ période de polarité \\ déplacement des pôles \\ déplacement polaire \\ auto-inversion du magnétisme rémanent \\ magnétisation rémanente \\ expansion du fond des mers \\ zone de subduction \\ faille transformante \\ fossé \\ voir polar wandering
}

\section{BIBLIOGRAPHIE}

COURTILLOT, Vincent, les "Panaches" moteurs de la dérive des continents, dans La Recherche, mars 1973 , vol. $4, \mathrm{n}^{\circ} 32$, p. 270-272.

DUNCAN, R. A., Mantle Plumes, Movement of the European Plate, and Polar Wandering, dans Nature, 8 septembre 1972 , vol. $239, \mathrm{n}^{\circ} 5367$, p. 82-86.

GARY, Margaret, Glossary of Geology, Washington (D.C.), American Geological Institute, $1972,805 \mathrm{p}$.

HALLAM, A.; Alfred Wegener and the Hypothesis of Continental Drift, dans Scientific American, février 1975 , vol. $232, \mathrm{n}^{\circ} 2, \mathrm{p} .88-97$.

HEEZEN, Bruce C. and Ian D. MACGREGOR, The Evolution of the Pacific, dans Scientific American, novembre 1973 , vol. $229, \mathrm{n}^{\circ} 5$, p. 102-112. JAMES, David E., The Evolution of the Andes, dans Scientific American, août 1973, vol. 229,
n $^{\circ}$ 2, p. 61-69.

MORGAN, W. J., Convection Plumes in the Lower Mantle, dans Nature, 5 mars 1971, vol. $230, \mathrm{n}^{\circ} 5288$, p. $42-43$.

ROUBAULT, Marcel, la Dérive des continents, Paris, "Que sais-je ? ", $\mathrm{n}^{\circ}$ 1503, P.U.F., $1972,126 \mathrm{p}$.

UYEDA, Seiya, Dérive des continents et tectonique des plaques, dans La Recherche, juilletaoût 1972 , vol. $3, \mathrm{n}^{\circ} 25$, p. 649-664.

VALENTINE, James W, and Eldridge M. MOORES, Plate Tectonics and the History of Life in the Oceans, dans Scientific American, avril 1974, vol. 230, n 4, p. 80-89.

WHITTEN, D. G.A., The Penguin Dictionary of Geology, Hammondsworth (England), Penguin Books Ltd., 1972, $495 \mathrm{p}$.

WYLLIE, Peter J., The Earth's Mantle, dans Scientific American, mars 1975, vol. 232, $\mathrm{n}^{\circ} 3$, p. 50-63. 